\title{
Fully flexible dye-sensitized solar cells photoanode modified with titanium dioxide- graphene quantum dot light scattering layer
}

\begin{abstract}
Herein, we report the preparation of titanium dioxide-graphene quantum dot (TG) as a light scattering layer (LSL) using facile electrodeposition and drop-casting. A fully flexible DSSC device containing TG LSL was capable to generate efficiency of $5.18 \%$ which is $95 \%$ higher than the bare photoanode (2.65\%). Upon the inclusion of TG LSL, the vast enhancement of efficiency was observed which attributed to the improvement in dye loading capacity and light scattering ability. The fully flexible DSSC also depicted higher charge collection efficiency compared to the bare photoanode, indicating enhanced light scattering ability.
\end{abstract}

Keyword: Dye-sensitized solar cell; Titanium dioxide-graphene quantum dot; Electrodeposition; Light scattering layer; Fully flexible DSSC device 